\title{
Evaluation of the Pharmacokinetics and Pharmacodynamics of Prasugrel in Japanese Elderly Subjects
}

\author{
Tomoko Hasunuma $^{1,2,3} \cdot$ Hiroyuki Fukase $^{4} \cdot$ Atsuhiro Miyazaki $^{5} \cdot$ Yasuhiro Nishikawa $^{6}$
}

Published online: 17 April 2017

(C) The Author(s) 2017. This article is an open access publication

\begin{abstract}
Background and Objective An increased incidence in bleeding events has been reported in Western elderly patients receiving prasugrel. Therefore, doses in Japanese elderly subjects need to be carefully determined. We assessed the pharmacokinetic and pharmacodynamic effects of prasugrel at the clinical dose used in Japan in healthy Japanese elderly subjects compared with nonelderly subjects.

Methods In an open-label parallel-group study conducted in Japan, two groups (elderly, aged $>75$ years; non-elderly, aged $45-65$ years) received a $20-\mathrm{mg}$ loading dose and a 3.75-mg maintenance dose of prasugrel for 7 days. Plasma concentration of its active metabolite, R-138727, and pharmacokinetic parameters were determined on days 1 and 7 after dosing. Pharmacodynamic response to $20 \mu \mathrm{M}$
\end{abstract}

Electronic supplementary material The online version of this article (doi:10.1007/s40261-017-0525-0) contains supplementary material, which is available to authorized users.

Atsuhiro Miyazaki

miyazaki.atsuhiro.f3@daiichisankyo.co.jp

1 Kitasato University Research Center for Clinical Pharmacology, Tokyo, Japan

2 Department of Research, Clinical Trial Center, Kitasato University Kitasato Institute Hospital, Tokyo, Japan

3 Kitasato Clinical Research Center (KCRC), Kitasato University School of Medicine, Tokyo, Japan

4 CPC Clinical Trial Hospital, Medipolis Medical Research Institute, Kagoshima, Japan

5 Clinical Development Department, Daiichi Sankyo Co., Ltd., 1-2-58 Hiromachi, Shinagawa-ku, Tokyo 140-8710, Japan

6 Safety and Risk Management Department, Daiichi Sankyo Co., Ltd., Tokyo, Japan of adenosine diphosphate-induced platelet aggregation was measured by light transmission aggregometry.

Results A total of 47 subjects were enrolled (23 elderly, 24 non-elderly). There was no statistically significant difference in pharmacokinetic parameters between groups: area under the plasma concentration-time curve up to the last quantifiable time and maximum plasma concentration were about 174-175 $\mathrm{ng} \cdot \mathrm{h} / \mathrm{mL}$ and $134-153 \mathrm{ng} / \mathrm{mL}$, respectively, after the loading dose; and about $25-26 \mathrm{ng} \cdot \mathrm{h} / \mathrm{mL}$ and $25 \mathrm{ng} / \mathrm{mL}$, respectively, after the maintenance dose. Inhibition of platelet aggregation was higher in the elderly subjects than in the non-elderly subjects, with a statistically significant difference from $24 \mathrm{~h}$ after the loading dose. No serious adverse events (bleeding or non-bleeding) occurred.

Conclusions Prasugrel (20-mg loading dose; 3.75-mg maintenance dose) produced a slight increase in antiplatelet efficacy in elderly compared with non-elderly subjects, despite no statistically significant difference in the pharmacokinetics.

\section{Key Points}

Prasugrel at a loading dose of $20 \mathrm{mg}$ and a maintenance dose of $3.75 \mathrm{mg}$ was administered to healthy Japanese elderly and non-elderly subjects. No statistically significant difference was found between the two groups in the pharmacokinetic parameters; however, the antiplatelet effect was higher in elderly than in non-elderly subjects with a statistically significant difference from $24 \mathrm{~h}$ after dosing.

No serious or clinically severe adverse events were observed in either elderly or non-elderly subjects. 


\section{Introduction}

Prasugrel, a thienopyridine prodrug, is metabolized in vivo to an active metabolite, R-138727, which binds to $\mathrm{P}_{2} \mathrm{Y}_{12}$ receptors irreversibly and inhibits adenosine diphosphate (ADP)-induced platelet aggregation [1-3]. Prasugrel is a potent inhibitor of platelet aggregation, with a rapid onset of antiplatelet effect and minimal inter-individual variability compared with clopidogrel [4-7]. In patients undergoing percutaneous coronary intervention, the TRITON-TIMI 38 (Trial to Assess Improvement in Therapeutic Outcomes by Optimizing Platelet Inhibition with Prasugrel-Thrombolysis in Myocardial Infarction 38) study demonstrated that prasugrel significantly reduced the primary endpoint of myocardial infarction, stroke, and cardiovascular death compared with clopidogrel [8]. Prasugrel (60-mg loading dose and 10-mg maintenance dose) is used for the reduction of atherothrombotic cardiovascular events in patients undergoing percutaneous coronary intervention in USA, the European Union, and in other countries.

In the TRITON-TIMI 38 [8], the incidence of bleeding events was increased in elderly patients; however, in that study, the dose of prasugrel (60-mg loading dose and 10-mg maintenance dose) was higher than the approved dose in Japan (20-mg loading dose and 3.75-mg/day maintenance dose), and also aspirin was co-administered. The evaluation of the effect of prasugrel in elderly Japanese subjects is very important because bleeding risk in general is increased with aging [9-12]. In the present study, we compared the pharmacokinetics, pharmacodynamics, and safety of prasugrel monotherapy (20-mg loading dose and $3.75-\mathrm{mg} /$ day maintenance dose) in healthy Japanese elderly and non-elderly subjects.

\section{Methods}

\subsection{Subjects and Study Design}

This study was conducted as an open-label parallel-group study to compare the pharmacokinetics and pharmacodynamics between elderly (aged $>75$ years) and non-elderly healthy Japanese (aged 45-65 years) subjects at the Kitasato University Research Center for Clinical Pharmacology and CPC Clinical Trial Hospital. The study included subjects with a body mass index of $\geq 18.5$ and $<30.0 \mathrm{~kg} / \mathrm{m}^{2}$ and with blood pressure $<150 / 95 \mathrm{mmHg}$. Subjects who met any of the following criteria were excluded from the study: those with a current or past history of a disorder of the central nervous system, circulatory system, respiratory system, hematological or hematopoietic function, digestive system, liver function or kidney function; those with grossly evident petechial hemorrhage; those with a bleeding diathesis or bleeding disorder; those with body weight $<50 \mathrm{~kg}$; and those with laboratory test values exceeding the institutional standard value.

For those who agreed to participate in the present study, investigators performed the screening test during the period between 2 and 30 days before the first dose of the study drug, and for those who were judged eligible, subsequent screening tests were performed both on the day before and on the day of the first dose. Subjects received the 20-mg loading dose of prasugrel on the first day. From days 2 to 7 , they received the $3.75-\mathrm{mg} /$ day maintenance dose.

The present study was conducted in accordance with the Declaration of Helsinki in compliance with Good Clinical Practice and with the approval of the institutional review board. Written informed consent to participate in the study was obtained from subjects before their participation in the study.

\subsection{Pharmacokinetics}

Blood samples were collected before dosing and at 0.25 , $0.5,1,1.5,2,4,6,8,12$, and $24 \mathrm{~h}$ post-dose on day 1 , and before dosing and at $0.25,0.5,1,1.5,2,4,6$, and $8 \mathrm{~h}$ postdose on day 7 for determination of the plasma concentration of R-138727 (the active metabolite of prasugrel). The analysis was performed using a liquid chromatographytandem mass spectrometry method with the lower quantification limit of $0.5 \mathrm{ng} / \mathrm{mL}$.

A 5-mL venous blood sample was taken from the antebrachial cutaneous vein of each subject (using a vacuum blood collection tube with EDTA-2K) for all collection points. A $25-\mu \mathrm{L}$ of $3^{\prime}$-methoxyphenacyl bromide acetonitrile solution was added to each sample, which was mixed by inversion and chilled on ice. After centrifugation of the sample ( $3000 \mathrm{rpm}$ for $10 \mathrm{~min}$ at $4{ }^{\circ} \mathrm{C}$ ), the obtained plasma was divided into two storage containers (about $1.0 \mathrm{~mL}$ each) and sent for drug concentration analysis at a different facility. Component analysis was performed on an Inertsi ${ }^{\circledR}$ ODS-3 high-performance liquid chromatography column (GL Sciences Inc., Torrance, CA, USA) using methanol and $1 \%$ formic acid for the mobile phase (flow rate, $0.25 \mathrm{~mL} / \mathrm{min}$; injection volume, $5 \mu \mathrm{L}$ ). For mass spectrometry an API4000 quadrupole tandem mass spectrometer (AB SCIEX, Framingham, MA, USA) was used. For chromatogram analysis, the software Analyst, Version 1.4.1 (Applied biosystems/MDS SCIEX, Foster City, CA, USA) program was used to calculate the peak area ratio (Y) for R-138727. The calibration curve was derived by linear regression analysis using the preparation 
concentration $(X)$ with reweighting $\left(1 / X^{2}\right)$; internal standards were employed to achieve quantitative calculations. The linearity of the calibration curve was confirmed by correlation coefficient $(r) \geq 0.9800$. The acceptable relative error for values obtained by back-calculation was considered to be $\pm 15 \%$ for data above the lower limit of quantification, and between -15.0 and $20.0 \%$ for data below the lower limit of quantification.

The following pharmacokinetic parameters were calculated using WinNonlin-Professional, Version 5.2 (Pharsight Corporation, Mountain View, CA, USA): area under the plasma concentration-time curve up to infinity, area under the plasma concentration-time curve up to the last quantifiable time, area under the plasma concentrationtime curve during a dosing interval, maximum plasma concentration, time to reach maximum plasma concentration, and terminal elimination half-life.

For the comparison of pharmacokinetic parameters, logtransformed values of each parameter (for time to reach maximum plasma concentration, untransformed values) were used, and the ratios of geometric means (for time to reach maximum plasma concentration, differences in the arithmetic means) and $90 \%$ confidence interval (CI) between each age group were calculated. If the $90 \%$ CI did not contain 1 , the results were considered statistically significant.

\subsection{Pharmacodynamics}

Blood samples were collected on day 1 (before dosing, and at $1,4,8$, and $24 \mathrm{~h}$ post-dose), and day 7 (before dosing, and at 4 and $8 \mathrm{~h}$ post-dose). For all timepoints, a $4.5-\mathrm{mL}$ venous blood sample was taken from the antebrachial cutaneous vein of each subject (using a vacuum blood collection tube with $3.8 \%$ sodium citrate). The pharmacodynamic measurements were determined by the light transmission method in response to 5 and $20 \mu \mathrm{M}$ of ADP. Inhibition of platelet aggregation (IPA) at each blood sampling timepoint was calculated using the following formula:

$\operatorname{IPA}(\%)$

$=\frac{\text { MPA at screening }- \text { MPA at each measurement point }}{\text { MPA at screening }} \times 100$,

where MPA is the maximum platelet aggregation.

For IPA obtained at each blood sampling point for each subject, the arithmetic mean was calculated. The differences in the arithmetic mean IPA and two-sided 90\% CI between the elderly and non-elderly groups were used to compare the inhibitory effect of prasugrel in the two groups. If the $90 \% \mathrm{CI}$ did not contain 0 , the results were considered statistically significant.

\subsection{Safety}

The safety of prasugrel was evaluated by assessing the incidence of adverse events (AEs) from the time of administration of the study drug until the follow-up test conducted 9-15 days after the last dosing. An AE was defined as the occurrence or worsening of subjective or objective symptoms, or an abnormal change in laboratory test values, in a subject who received prasugrel, which does not necessarily have a causal relationship with the drug. Adverse events were graded according to their severity, as mild, moderate, and severe. The following were considered serious AEs: (1) death, (2) conditions potentially leading to death, (3) conditions requiring hospitalization or prolonged hospitalization for their treatment, (4) disability, (5) conditions potentially leading to disability, (6) conditions similar to $1-5$ in severity, and (7) congenital disease or abnormality in a later generation. Hemorrhagic events were considered important AEs in the present study.

\subsection{Statistical Analysis}

Based on previous clinical studies, we estimated the standard deviations of IPA as 8 and $10 \%$, and the difference in the arithmetic mean IPA between the elderly and nonelderly groups as $10 \%$. Under these assumptions, we twice performed a $t$ test with a one-sided significance level of 0.05 for 40 subjects; we confirmed high statistical power (97.9 and $87.5 \%)$. Additionally, even if there were two dropouts, the statistical power would still be adequate, at 97.3 and $85.5 \%$, respectively. Consequently, the number of subjects was determined as 40 . The time course of the plasma concentration of R-138727 was plotted. Pharmacokinetic parameters were estimated by non-compartmental analysis methods.

Patient demographical characteristics were evaluated as follows: for categorical variables, frequency tables by age were generated. For quantitative variables, summary statistics (number of subjects, arithmetic means, standard deviations, and median/minimum/maximum values) were calculated. SAS System Release, Version 8.2 (SAS Institute Inc., Cary, NC, USA) was used for data collection and analysis.

\section{Results}

\subsection{Disposition of Subjects}

As shown in Fig. 1, 120 subjects were prescreened and then 57 subjects were screened for eligibility; 47 subjects were subsequently enrolled ( 23 elderly and 24 non-elderly) and received the study drug. However, one subject in the 


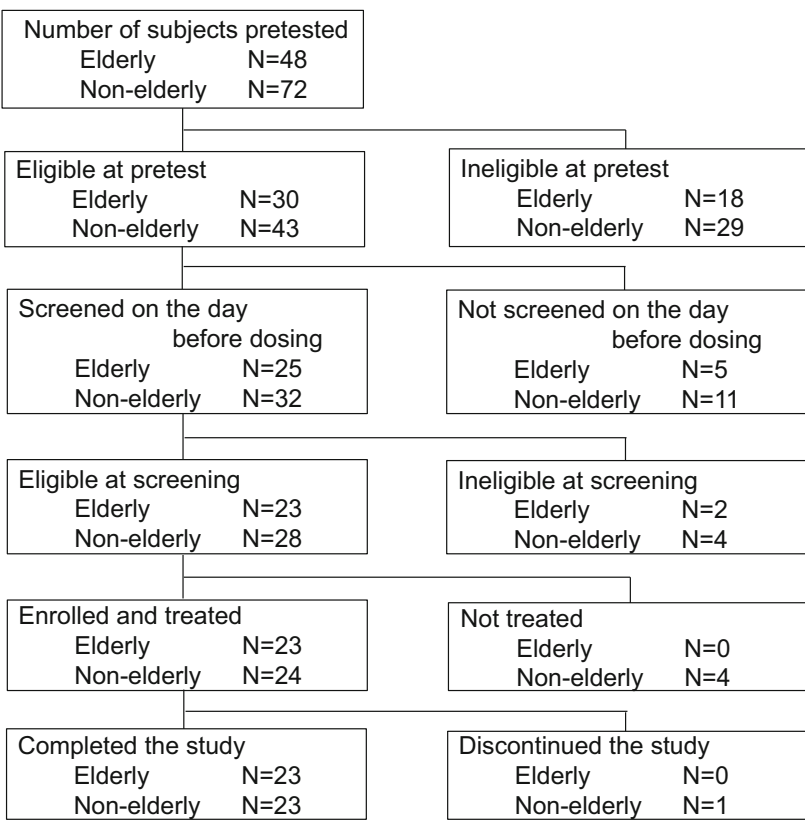

Fig. 1 Patient flow diagram

non-elderly group discontinued the study drug because of an AE, fecal occult blood, on day 2 after dosing.

Table 1 shows the baseline characteristics of the subjects. The mean age of the elderly group was $77.6 \pm 2.9$ years and the mean age of the non-elderly group was $58.2 \pm 5.5$ years. The proportion of current smokers was 8.7 and $29.2 \%$ in the elderly and non-elderly groups, respectively. There was no statistically significant difference in the other baseline characteristics between the two groups.

Table 1 Baseline characteristics of subjects

\begin{tabular}{lll}
\hline Characteristic & Elderly & Non-elderly \\
\hline Number of subjects $_{\text {Age }(\text { years })^{\text {a }}}$ & 23 & 24 \\
Median & $77.6 \pm 2.9$ & $58.2 \pm 5.5$ \\
Minimum, maximum & 77.0 & 60.0 \\
Body weight $(\mathrm{kg})^{\mathrm{a}}$ & 75,86 & 48,64 \\
Median & $58.8 \pm 8.0$ & $60.4 \pm 7.5$ \\
Minimum, maximum & 57.5 & 59.6 \\
BMI $\left(\mathrm{kg} / \mathrm{m}^{2}\right)^{\mathrm{a}}$ & $50.1,83.6$ & $50.8,78.1$ \\
Median & $24.0 \pm 2.8$ & $23.5 \pm 3.0$ \\
Minimum, maximum & 24.7 & 23.5 \\
Male sex $[n(\%)]$ & $20.0,29.7$ & $18.7,29.7$ \\
Smoking habits $[n(\%)]$ & $14(60.9)$ & $15(62.5)$ \\
Non-smoker & & \\
Current smoker & $17(73.9)$ & $15(62.5)$ \\
Ex-smoker & $2(8.7)$ & $7(29.2)$ \\
\hline
\end{tabular}

$B M I$ body mass index

${ }^{\text {a }}$ Values are expressed as the mean \pm standard deviation, unless otherwise noted

\subsection{Pharmacokinetics}

The plasma concentration of R-138727 increased rapidly after administration in both groups, reached a maximum at 30 min after administration, and then decreased rapidly, both on days 1 and 7 (Fig. 2a, b, respectively). The plasma concentration curves were almost superimposable between elderly and non-elderly subjects at $1.5 \mathrm{~h}$ after dosing. By $24 \mathrm{~h}$ after the loading dose, or $8 \mathrm{~h}$ after the maintenance dose, the plasma concentration was near the lower quantifiable limit in both groups.

The pharmacokinetic parameters of R-138727 are shown in Table 2. The area under the plasma concentration-time curve up to the last quantifiable time values, as expressed as geometric mean (percentage coefficient of variation), in elderly and non-elderly subjects were 173.5 (34.7) $\mathrm{ng} \cdot \mathrm{h} / \mathrm{mL}$ and 174.5 (35.9) $\mathrm{ng} \cdot \mathrm{h} / \mathrm{mL}$, respectively, after the loading dose (day 1), and $26.0(31.2) \mathrm{ng} \cdot \mathrm{h} / \mathrm{mL}$ and 24.7 (38.8) $\mathrm{ng} \cdot \mathrm{h} / \mathrm{mL}$, respectively, after the maintenance dose (day 7). Maximum plasma concentration values in elderly and non-elderly subjects were $134.3(62.1) \mathrm{ng} / \mathrm{mL}$ and $153.3(62.1) \mathrm{ng} / \mathrm{mL}$, respectively, on day 1 , and 25.2 (44.7) $\mathrm{ng} / \mathrm{mL}$ and $24.9(68.7) \mathrm{ng} / \mathrm{mL}$, respectively, on day 7. The estimated ratios of the geometric mean values for
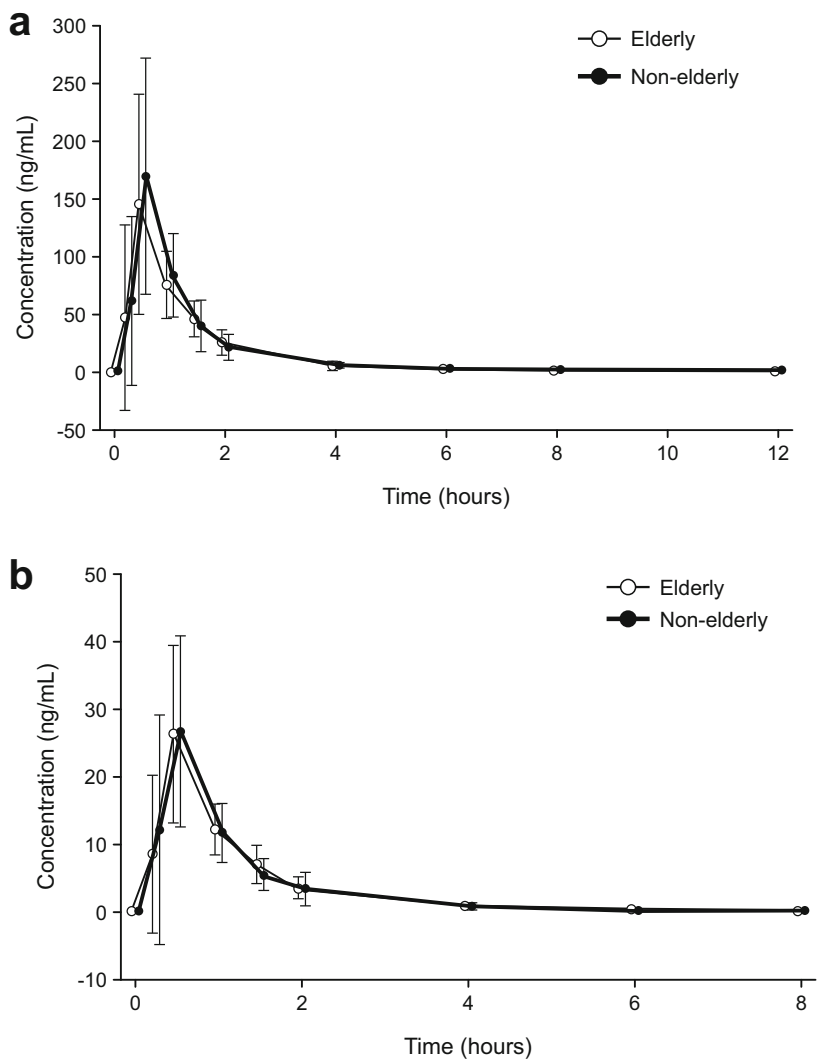

Fig. 2 Plasma concentrations of R-138727, the active metabolite of prasugrel, in elderly and non-elderly subjects on $\mathbf{a}$ day 1 and $\mathbf{b}$ day 7 . Data are expressed as the arithmetic mean \pm standard deviation 
Table 2 Pharmacokinetic parameters for R-138727, the active metabolite of prasugrel

\begin{tabular}{|c|c|c|c|}
\hline & Elderly $(n=23)$ & Non-elderly $(n=23)$ & Ratio $^{\mathrm{a}}(90 \% \mathrm{CI})$ \\
\hline \multicolumn{4}{|l|}{ Day 1} \\
\hline $\mathrm{AUC}_{\text {last }}(\mathrm{ng} \cdot \mathrm{h} / \mathrm{mL})$ & $173.5(34.7)$ & $174.5(35.9)$ & $0.994(0.837$ to 1.181$)$ \\
\hline $\mathrm{AUC}_{0-\mathrm{inf}}(\mathrm{ng} \cdot \mathrm{h} / \mathrm{mL})$ & $179.0(34.6)$ & $178.8(36.1)$ & $1.001(0.843$ to 1.189$)$ \\
\hline$C_{\max }(\mathrm{ng} \cdot \mathrm{h} / \mathrm{mL})$ & $134.3(62.1)$ & $153.3(62.1)$ & $0.876(0.658$ to 1.166$)$ \\
\hline$t_{\max }(\mathrm{h})$ & $0.57(0.27)$ & $0.59(0.23)$ & $-0.019(-0.145$ to 0.107$)$ \\
\hline$t_{1 / 2}(\mathrm{~h})$ & $4.4(60.9)$ & $3.5(90.3)$ & $1.262(0.903$ to 1.763$)$ \\
\hline \multicolumn{4}{|l|}{ Day 7} \\
\hline $\mathrm{AUC}_{\text {last }}(\mathrm{ng} \cdot \mathrm{h} / \mathrm{mL})$ & $26.0(31.2)$ & $24.7(38.8)$ & $1.055(0.891$ to 1.250$)$ \\
\hline $\mathrm{AUC}_{\text {tau }}(\mathrm{ng} \cdot \mathrm{h} / \mathrm{mL})$ & $27.1(30.6)$ & $26.2(36.2)$ & $1.033(0.878$ to 1.216$)$ \\
\hline$C_{\max }(\mathrm{ng} / \mathrm{mL})$ & $25.2(44.7)$ & $24.9(68.7)$ & $1.011(0.777$ to 1.317$)$ \\
\hline$t_{\max }(\mathrm{h})$ & $0.59(0.28)$ & $0.61(0.36)$ & $-0.022(-0.181$ to 0.138$)$ \\
\hline$t_{1 / 2}(\mathrm{~h})$ & $1.0(65.7)$ & $0.8(50.5)$ & $1.176(0.896$ to 1.544$)$ \\
\hline
\end{tabular}

Values are geometric mean (percentage coefficient of variation). $t_{\max }$ is expressed as arithmetic mean (standard variation)

$A U C_{\text {last }}$ area under the plasma concentration-time curve from time 0 to last measurement, $A U C_{\text {tau }}$ area under the plasma concentration-time curve over a dosing interval, $A U C_{0-i n f}$ area under the plasma concentration-time curve up to infinity, $C I$ confidence interval, $C_{\max }$ maximum plasma concentration, $t_{\max }$ time to $C_{\max }, t_{1 / 2}$ terminal elimination half-life

${ }^{a}$ Ratios were the value in elderly subjects/value in the non-elderly subjects area under the plasma concentration-time curve up to the last quantifiable time between elderly and non-elderly subjects (as calculated by the value in elderly subjects/value in non-elderly subjects) and its $90 \%$ CI were $0.994(0.837-1.181)$ after the loading dose and 1.055 (0.891-1.250) after the maintenance dose, showing no statistically significant difference between the two groups. The ratios for maximum plasma concentration also showed no significant difference for both groups after the loading dose [0.876 (0.658-1.166)] and maintenance dose [1.011 $(0.777-1.317)]$. Taken together, these data indicated that there was no statistically significant difference in the pharmacokinetic parameters of elderly subjects compared with non-elderly subjects.

\subsection{Pharmacodynamics}

Prasugrel (in response to $20 \mu \mathrm{M}$ of ADP) inhibited platelet aggregation in both groups (Fig. 3). The IPA increased rapidly after the loading dose of prasugrel was administered, reaching a maximum of about $50 \%$ at $8 \mathrm{~h}$. The inhibitory effect was maintained without attenuation during the maintenance dose administration, with a maximum of about $40 \%$ at $4 \mathrm{~h}$.

The inhibitory effects on platelet aggregation between the elderly and non-elderly groups were not significantly different up to $8 \mathrm{~h}$ after the loading dose. However, the elderly group exhibited a greater inhibitory effect compared with the non-elderly group at $24 \mathrm{~h}$ after the loading dose, and at 4 and $8 \mathrm{~h}$ after the maintenance dose. The

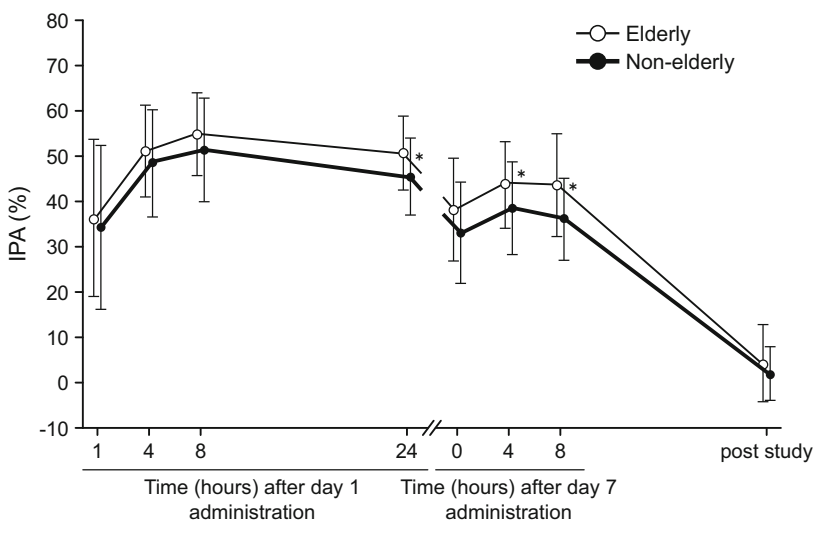

Fig. 3 Inhibition of adenosine diphosphate $(20 \mu \mathrm{M})$-induced platelet aggregation in elderly and non-elderly subjects. Data are expressed as the arithmetic mean \pm standard deviation. $* p<0.05$ vs non-elderly subjects. IPA inhibition of platelet aggregation

results of the comparison of IPA values are shown in Table 3 . The inhibitory effects on platelet aggregation with $5 \mu \mathrm{M}$ of ADP were similar to those with $20 \mu \mathrm{M}$ of ADP (Supplementary Table).

\subsection{Safety}

There were no serious AEs. In the non-elderly group, one subject discontinued the study drug because of fecal occult blood; this event was considered severe but it had resolved without any treatment by the following day. In addition, there were 11 fecal occult blood events (elderly group, seven subjects and non-elderly group, four subjects) and 
Table 3 Comparison of the inhibition of platelet aggregation values in the elderly and non-elderly groups

\begin{tabular}{|c|c|c|c|c|c|c|}
\hline \multirow[t]{2}{*}{ Time after dosing } & \multicolumn{2}{|c|}{ Elderly $(n=23)$} & \multicolumn{2}{|c|}{ Non-elderly $(n=23)$} & \multicolumn{2}{|l|}{ Comparison for both groups } \\
\hline & Mean $(\%)$ & $95 \% \mathrm{CI}$ & Mean $(\%)$ & $95 \% \mathrm{CI}$ & Difference in arithmetic mean & $90 \% \mathrm{CI}$ \\
\hline \multicolumn{7}{|l|}{ Day 1} \\
\hline $1 \mathrm{~h}$ & 36.3 & $28.7-44.0$ & 34.2 & 26.4 to 42.1 & 2.1 & -6.8 to 11.0 \\
\hline $4 \mathrm{~h}$ & 51.1 & $46.8-55.5$ & 48.5 & 43.4 to 53.6 & 2.6 & -2.8 to 8.0 \\
\hline $8 \mathrm{~h}$ & 54.8 & $50.8-58.8$ & 51.5 & 46.6 to 56.5 & 3.3 & -1.9 to 8.4 \\
\hline \multicolumn{7}{|l|}{ Day 2} \\
\hline Before dosing & 50.8 & $47.3-54.3$ & 45.6 & 41.9 to 49.3 & 5.2 & 1.1 to 9.3 \\
\hline \multicolumn{7}{|l|}{ Day 7} \\
\hline Before dosing & 38.2 & $33.3-43.1$ & 33.0 & 28.1 to 37.8 & 5.2 & -0.4 to 10.8 \\
\hline $4 \mathrm{~h}$ & 43.6 & $39.5-47.7$ & 38.5 & 34.1 to 42.9 & 5.1 & 0.24 to 10.0 \\
\hline $8 \mathrm{~h}$ & 43.6 & $38.7-48.5$ & 36.0 & 32.0 to 40.0 & 7.6 & 2.5 to 12.7 \\
\hline Post-study & 4.2 & $0.5-.9$ & 1.8 & -0.8 to 4.3 & 2.4 & -1.2 to 6.1 \\
\hline
\end{tabular}

Values are expressed as arithmetic mean

CI confidence interval

five subcutaneous bleeding events (elderly group, four subjects and non-elderly group, one subject). One oral hematoma and one retinal hemorrhage in the elderly group were also reported. All of the events were considered related to prasugrel treatment, which were transient and required no treatment.

\section{Discussion}

In the previous study of prasugrel, Umemura et al. showed that a single oral administration at a loading dose of up to $30 \mathrm{mg}$ and a multiple administration at a maintenance dose of up to $10 \mathrm{mg}$ /day were tolerated in healthy Japanese subjects [13]. In the present study, we used prasugrel at the approved dose in Japan (20-mg loading dose and $3.75-\mathrm{mg} /$ day maintenance dose) to compare the pharmacokinetic and pharmacodynamic profiles of prasugrel in healthy Japanese elderly subjects and nonelderly subjects. We found that in the elderly subjects, prasugrel had a higher antiplatelet effect, with a statistically significant difference from $24 \mathrm{~h}$ after dosing, despite no significant difference in the pharmacokinetic parameters, compared with non-elderly subjects. No serious or clinically severe AEs were observed in either elderly or non-elderly subjects.

In the baseline characteristics, there was a higher ratio of smokers in the non-elderly subjects. The TRILOGY ACS trial [14] showed that the $\mathrm{P}_{2} \mathrm{Y}_{12}$ reaction unit tends to be lower in smokers than in non-smokers. Therefore, we consider that the higher antiplatelet effect observed in the eldery subjects was not caused by the difference in the baseline characteristics.
In a study in the UK [15] comparing the pharmacokinetics and pharmacodynamics of prasugrel (maintenance dose $5 \mathrm{mg}$ /day for 10 days followed by $10 \mathrm{mg} /$ day for 10 days in combination with aspirin $75 \mathrm{mg}$ ) between elderly (aged 65-80 years) and non-elderly (aged 20-39 years) subjects, no difference was found in the pharmacokinetic parameters. However, the maximum platelet aggregation was higher in the elderly subjects; the finding was inconsistent with the results of the pharmacodynamics in the present study. Therefore, it appears that prasugrel does not consistently cause increased pharmacodynamic effects in elderly subjects.

In the PRASFIT-ACS study, a phase III registration study in Japan that compared prasugrel and clopidogrel used in combination with aspirin in patients with acute coronary syndrome who were scheduled to undergo percutaneous coronary intervention, the antiplatelet effect (measured by VerifyNow, Accumetrics, San Diego, CA, USA) in patients aged $\geq 75$ years was comparable to that in the overall study population, according to the subgroup analysis. In addition, the incidence of clinically significant bleeding events was similar to that of clopidogrel [16]. In the present study, there were no serious or clinically severe AEs in the elderly group.

\section{Conclusion}

Administration of prasugrel at a 20-mg loading dose and a 3.75-mg maintenance dose in healthy Japanese elderly subjects showed no statistically significant difference in the pharmacokinetics but a higher antiplatelet effect, with a statistically significant difference from $24 \mathrm{~h}$ after dosing, 
compared with in non-elderly subjects. No serious or clinically severe AEs were observed.

Acknowledgements The authors thank the study participants, investigators, and supporting staff for their cooperation.

\section{Compliance with Ethical Standards}

Funding The present study was sponsored by Daiichi Sankyo Co., Ltd. (Tokyo, Japan).

Conflict of Interest AM and YN are employees of Daiichi Sankyo Co., Ltd. TH and HF have no conflicts of interest associated with the work presented in this manuscript.

Ethics Approval All procedures in this study were in accordance with the Declaration of Helsinki in compliance with Good Clinical Practice and with the approval of the institutional review board.

Consent to Participate Written informed consent to participate in the study was obtained from subjects before their participation in the study.

Open Access This article is distributed under the terms of the Creative Commons Attribution-NonCommercial 4.0 International License (http://creativecommons.org/licenses/by-nc/4.0/), which permits any noncommercial use, distribution, and reproduction in any medium, provided you give appropriate credit to the original author(s) and the source, provide a link to the Creative Commons license, and indicate if changes were made.

\section{References}

1. Niitsu Y, Jakubowski JA, Sugitachi A, Asai F. Pharmacology of CS-747 (prasugrel, LY640315), a novel potent antiplatelet agent with in vivo P2Y12 receptor antagonist activity. Semin Thromb Hemost. 2005;31:184-94.

2. Hasegawa M, Sugitachi A, Ogawa T, et al. Stereoselective inhibition of human platelet aggregation by R-138727, the active metabolite of CS-747 (prasugrel, LY640315), a novel P2Y12 receptor inhibitor. Thromb Haemost. 2005;94:593-8.

3. Rehmel JL, Eckstein JA, Farid NA, et al. Interactions of two major metabolites of prasugrel, a thienopyridine antiplatelet agent, with the cytochromes P450. Drug Metab Dispos. 2006;34:600-7.

4. Jakubowski JA, Winters KJ, Naganuma H, et al. Prasugrel: a novel thienopyridine antiplatelet agent. A review of preclinical and clinical studies and the mechanistic basis for its distinct antiplatelet profile. Cardiovasc Drug Rev. 2007;25:357-74.

5. Brandt JT, Payne CD, Wiviott SD, et al. A comparison of prasugrel and clopidogrel loading doses on platelet function: magnitude of platelet inhibition is related to active metabolite formation. Am Heart J. 2007;153(66):e9-16.

6. Jernberg T, Payne CD, Winters KJ, et al. Prasugrel achieves greater inhibition of platelet aggregation and a lower rate of nonresponders compared with clopidogrel in aspirin treated patients with stable coronary artery disease. Eur Heart J. 2006;27:1166-73.

7. Wiviott SD, Trenk D, Frelinger AL, et al. Prasugrel compared with high loading- and maintenance-dose clopidogrel in patients with planned percutaneous coronary intervention: the Prasugrel in Comparison to Clopidogrel for Inhibition of Platelet Activation and Aggregation-Thrombolysis in Myocardial Infarction 44 trial. Circulation. 2007;116:2923-32.

8. Wiviott SD, Braunwald $\mathrm{E}, \mathrm{McCabe} \mathrm{CH}$, et al. Prasugrel versus clopidogrel in patients with acute coronary syndromes. N Engl J Med. 2007;357:2001-15.

9. Manoukian SV. Predictors and impact of bleeding complications in percutaneous coronary intervention, acute coronary syndromes, and ST-segment elevation myocardial infarction. Am J Cardiol. 2009;104:9C-15C.

10. Feit F, Voeltz MD, Attubato MJ, et al. Predictors and impact of major hemorrhage on mortality following percutaneous coronary intervention from the REPLACE-2 trial. Am J Cardiol. 2007;100:1364-9.

11. Manoukian SV, Voeltz MD, Eikelboom J. Bleeding complications in acute coronary syndromes and percutaneous coronary intervention: predictors, prognostic significance, and paradigms for reducing risk. Clin Cardiol. 2007;30(10 Suppl 2):II-24-34.

12. Lopes RD, Alexander KP, Manoukian SV, et al. Advanced age, antithrombotic strategy, and bleeding in non-ST-segment elevation acute coronary syndromes. J Am Coll Cardiol. 2009;53:1021-30.

13. Umemura K, Ikeda Y, Kondo K. Pharmacokinetics and pharmacodynamics of prasugrel in healthy Japanese subjects. Drug Metab Pharmacokinet. 2016;31:285-91.

14. Cornel JH, Ohman EM, Neely B, et al. Impact of smoking status on platelet function and clinical outcomes with prasugrel vs. clopidogrel in patients with acute coronary syndromes managed without revascularization: insights from the TRILOGY ACS trial. Am Heart J. 2014;168(76-87):e1.

15. Small DS, Wrishko RE, Ernest CS 2nd, et al. Effect of age on the pharmacokinetics and pharmacodynamics of prasugrel during multiple dosing: an open-label, single-sequence, clinical trial. Drugs Aging. 2009;26:781-90.

16. Efient ${ }^{\circledR}$ tablets $3.75 \mathrm{mg} / 5 \mathrm{mg}$. Common technical document, published by Daiichi Sankyo Co., Ltd., Tokyo, Japan (in Japanese). 